\title{
Ru-catalyzed dehydrogenative coupling of carboxylic acids and silanes - a new method for the preparation of silyl ester
}

\author{
Guo-Bin Liu ${ }^{*}$ and Hong-Yun Zhao
}

\section{Preliminary Communication}

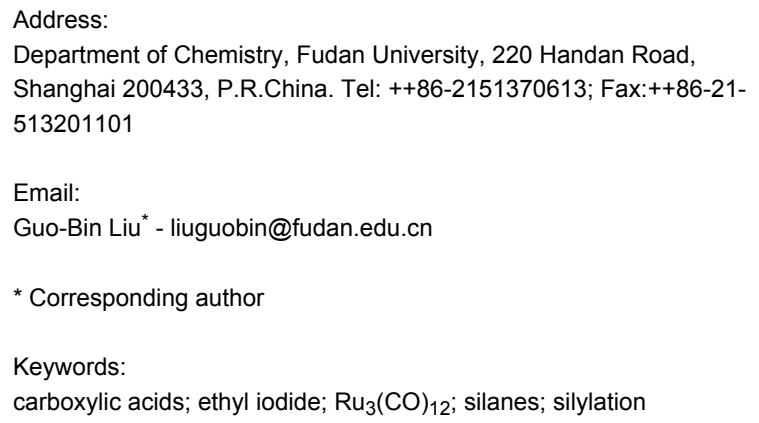

Beilstein Journal of Organic Chemistry 2008, 4, No. 27 doi:10.3762/bjoc. 4.27

Received: 21 May 2008 Accepted: 23 July 2008 Published: 30 July 2008

(c) 2008 Liu et al; licensee Beilstein-Institut. License and terms: see end of document.

\begin{abstract}
$\mathrm{Ru}_{3}(\mathrm{CO})_{12} / \mathrm{EtI}$ has been found to be an efficient catalyst system for the dehydrosilylation of carboxylic acids with silanes. In the presence of $1 \mathrm{~mol} \% \mathrm{Ru}_{3}(\mathrm{CO})_{12}$ and $4 \mathrm{~mol} \%$ EtI, dehydrosilylation reactions in toluene afforded the corresponding silyl esters at $100{ }^{\circ} \mathrm{C}$ in good and high yields.
\end{abstract}

\section{Introduction}

Polymers composed of nucleophilically-labile silyl ester bonds in the main chain are being studied as a new type of degradable functional polymers with the potential for an extremely broad range of degradation behavior through variation in the functionalities attached to the silicon atom. In the design of degradable materials, the physical and mechanical properties must be considered for performance in serving the expected function, while degradation rate and degradation products are also very important. Since the lability of a silyl ester linkage is dramatically affected by the substituents attached to the silicon atom, poly(silyl ester)s were found to be an ideal family of degradable polymers [1]. Also, multifunctional silyl esters have been found to be ideal cross-linking agents since they require only mild reaction conditions, especially for silicone elastomers. The demand for degradable poly(silyl ester)s has been increasing greatly due to biomedical field and environmental concerns [2-4]. Obviously, silyl esters are very important intermediates for the preparation of easily degradable functional poly(silyl ester)s, widely utilized as gene delivery carriers, matrices for drug delivery, biodegradable surgical devices, and recyclable materials [2-12]. To develop simple, economical and practical protocols for the conversion of carboxylic acids into silyl esters is not only required in normal organic synthesis procedure, but is also a prerequisite for the accurate performance of gas-chromatographic analyses in organic and biological chemistry [13, 14]. From the viewpoint of synthetic chemistry, the ideal protecting group for an active-hydrogen moiety such as carboxylic acid should be attached in high yield, be stable towards severe reaction conditions and, at the same time, be selectively removable in the presence of other functional groups 
carrying different protecting groups. Indeed, silylation of carboxylic acids is a useful method for their protection because deprotection of silyl esters is easily achieved under mild reaction conditions [15-18].

Generally, silyl esters are made by the coupling of carboxylic acids and chlorosilanes [19-24]. Unavoidably, $\mathrm{HCl}$ is formed in these procedures, and a stoichiometric or even an excess amount of bases such as amines or ammonia is needed to consume the $\mathrm{HCl}$ gas formed. Since chlorosilanes themselves are produced by the chlorination of silanes, either with chlorine gas [7] or with hydrochloric acid under $\mathrm{Pd} / \mathrm{C}$ catalysis [25,26], the synthesis of silyl esters from the corresponding silanes requires two reaction steps. Some newer synthetic protocols to silyl esters have been developed and a lot of literature focuses itself on the transition metal-catalyzed cross-coupling of an active hydrogen-moiety containing substances such as water and alcohols with silanes [27]. There are still few examples of dehydrogenative coupling reaction of carboxylic acids with silanes. Silylating agents such as allyltrimethylsilane, hexamethyldisilazane, aminosilanes, $N$-trimethylsilylalkanamines, $N$-trimethylsilyl-2-oxazolidinone, $N$-trimethylsilylacetamide, or trialkylsilyl 2-methallylprop-2-ene-1-sulfinates have been extensively utilized for the transformation of carboxylic acids into the desired silyl esters [28-43]. However, some shortcomings have been noted in these reported methods. The silylations of carboxylic acids with hexamethyldisilazane usually require prolonged reaction time under heating and continuous removal of ammonia or amine formed therein and the silylating agents are expensive. A few examples have been disclosed for dehydrosilylation reactions catalyzed by metal salts such as zinc chloride [26] or, more frequently, by transition metals and metal complexes such as $\left[\mathrm{CuH}\left(\mathrm{Ph}_{3} \mathrm{P}\right)\right]$ [34], $\mathrm{HPtCl}_{6}, \mathrm{Rh}$ and $\mathrm{Pd}$ [36-44], $\mathrm{Co}_{2}(\mathrm{CO})_{6}$ [45], $\mathrm{Cu}\left(\mathrm{Ph}_{3} \mathrm{P}\right)_{3} \mathrm{Cl}$ [46] and promoted by organocatalyst such as triphenylphosphine [47]. Generally, catalysts such as transition metals are expensive. $\left[\mathrm{CuH}\left(\mathrm{Ph}_{3} \mathrm{P}\right)\right]$ requires a multiple-step synthetic approach and in-situ generation protocols.

\section{Results and Discussion}

In this communication, we wish to report the first finding that a catalytic system of dodecacarbonyltriruthenium and ethyl iodide $\left[\mathrm{Ru}_{3}(\mathrm{CO})_{12} / \mathrm{EtI}\right]$ effectively promotes the dehydrogenative coupling of carboxylic acids with silanes, yielding the corresponding silyl esters selectively. The results are summarized in Scheme 1 and Table 1-Table 4.

Dehydrogenative coupling reactions were carried out by heating a mixture of carboxylic acid, silane and a catalytic amount of $\mathrm{Ru}_{3}(\mathrm{CO})_{12} / \mathrm{EtI}$ in solvents under a nitrogen atmosphere for several hours (Scheme 1, Table 1-Table 4, dehydrocoupling reaction was monitored by GC). The transformation of

Scheme 1: Dehydrogenative silyl ester synthesis with $\mathrm{Ru}_{3}(\mathrm{CO})_{12} / \mathrm{EtI}$.

\begin{tabular}{|c|c|c|c|c|c|c|}
\hline \multirow[t]{2}{*}{ Run } & \multirow[t]{2}{*}{$\mathrm{Ru}_{3}(\mathrm{CO})_{12}(\mathrm{~mol} \%)$} & \multirow[t]{2}{*}{ Etl $(\mathrm{mol} \%)$} & \multirow[t]{2}{*}{ Temp. $\left({ }^{\circ} \mathrm{C}\right)$} & \multirow[t]{2}{*}{ Time $(h)$} & \multicolumn{2}{|c|}{$\mathrm{GC}$ ratio $(\%)^{\mathrm{b}, \mathrm{c}}$} \\
\hline & & & & & $\mathrm{HSiEt}_{3}$ & $\mathrm{CH}_{3} \mathrm{CH}_{2} \mathrm{CO}_{2} \mathrm{SiEt}_{3}$ \\
\hline 1 & 0.25 & 4 & 100 & 12 & 66 & 34 \\
\hline 2 & 0.5 & 4 & 100 & 12 & 52 & 48 \\
\hline 3 & 1 & 4 & 100 & 8 & 0 & $100(95)$ \\
\hline 4 & 2 & 4 & 100 & 8 & 0 & $100(93)$ \\
\hline 5 & 4 & 4 & 100 & 8 & 0 & $100(94)$ \\
\hline 6 & 8 & 4 & 100 & 8 & 0 & $100(92)$ \\
\hline 7 & 1 & 8 & 100 & 8 & 0 & $100(92)$ \\
\hline 8 & 1 & 2 & 100 & 12 & 22 & 78 \\
\hline 9 & 1 & 4 & 20 & 24 & 100 & 0 \\
\hline 10 & 1 & 4 & 40 & 24 & 82 & 18 \\
\hline 11 & 1 & 4 & 60 & 24 & 70 & 30 \\
\hline 12 & 1 & 4 & 80 & 24 & 61 & 39 \\
\hline
\end{tabular}

apropionic acid $(20 \mathrm{mmol})$, triethylsilane $(20 \mathrm{mmol}) .{ }^{\mathrm{b}} \mathrm{GC}$ ratio. ${ }^{\mathrm{c}}$ lsolated yield in parentheses. 


\begin{tabular}{|c|c|c|c|c|}
\hline \multirow[t]{2}{*}{ Run } & \multirow[t]{2}{*}{ Solvent } & \multirow[t]{2}{*}{ Time (h) } & \multicolumn{2}{|c|}{ GC ratio (\%) } \\
\hline & & & $\mathrm{HSiEt}_{3}$ & $\mathrm{CH}_{3} \mathrm{CH}_{2} \mathrm{CO}_{2} \mathrm{SiEt}_{3}$ \\
\hline 1 & Toluene & 8 & 0 & 100 \\
\hline 2 & $n$-Octane & 24 & 22 & 78 \\
\hline 3 & Xylene & 24 & 17 & 83 \\
\hline 4 & Ethylbenzene & 24 & 15 & 85 \\
\hline 5 & tert-Butylbenzene & 24 & 14 & 86 \\
\hline 6 & Mesitylene & 24 & 12 & 88 \\
\hline 7 & Anisole & 24 & 14 & 86 \\
\hline 8 & Diethylene glycol diethyl ether & 24 & 20 & 80 \\
\hline 9 & DMF & 24 & 67 & 33 \\
\hline 10 & DMAc & 24 & 61 & 39 \\
\hline
\end{tabular}

\begin{tabular}{|c|c|c|c|c|}
\hline \multirow[t]{2}{*}{ Run } & \multirow[t]{2}{*}{ Catalyst } & \multirow[t]{2}{*}{ Time $(\mathrm{h})$} & \multicolumn{2}{|c|}{$\mathrm{GC}$ ratio $(\%)$} \\
\hline & & & $\mathrm{HSiEt}_{3}$ & $\mathrm{CH}_{3} \mathrm{CH}_{2} \mathrm{CO}_{2} \mathrm{SiEt}_{3}$ \\
\hline 1 & $\mathrm{Ru}_{3}(\mathrm{CO})_{12}$ & 8 & 0 & $100^{a}$ \\
\hline 2 & $\mathrm{Ru}_{3}(\mathrm{CO})_{12}$ & 8 & 9 & $91^{b}$ \\
\hline 3 & $\mathrm{Ru}_{3}(\mathrm{CO})_{12}$ & 24 & 81 & $19^{c}$ \\
\hline 4 & {$\left[\mathrm{RuCl}_{2}(\mathrm{CO})_{3}\right]_{2}$} & 24 & 9 & $11^{a}$ \\
\hline 5 & $\mathrm{RuCl}_{2}(\mathrm{CO})_{2}\left(\mathrm{PPh}_{3}\right)_{2}$ & 24 & 11 & $86^{a}$ \\
\hline 6 & $\mathrm{RuCl}_{2}\left(\mathrm{PPh}_{3}\right)_{3}$ & 24 & 10 & $84^{a}$ \\
\hline 7 & $\mathrm{Ru}(\mathrm{acac})_{3}$ & 24 & 27 & $73^{a}$ \\
\hline 8 & $\mathrm{RuCl}_{2}\left(2,2^{\prime} \text {-bipy }\right)_{3} 6 \mathrm{H}_{2} \mathrm{O}$ & 24 & 25 & $75^{a}$ \\
\hline
\end{tabular}

additive: 4 mol\% Etl, ${ }^{b}$ Additive: 4 mol\% EtBr, ${ }^{c}$ no Additive.

propionic acid with triethylsilane was employed as a model to optimize the reaction conditions.

The dehydrogenative coupling was found to be finished after 8 $\mathrm{h}$ at $100{ }^{\circ} \mathrm{C}$, in the presence of $1 \mathrm{~mol}^{\circ} \mathrm{Ru}_{3}(\mathrm{CO})_{12}$ and $4 \mathrm{~mol} \%$ EtI in toluene, giving the corresponding triethylsilyl propionate in $95 \%$ yield (Table 1, Run 3 ). When the amount of $\mathrm{Ru}_{3}(\mathrm{CO})_{12}$ was increased to 2, 4 or even $8 \mathrm{~mol} \%$, the product yields were 92-95\% (Table 1, Runs 4-6). The reaction proceeded more slowly, however, when the amount of $\mathrm{Ru}_{3}(\mathrm{CO})_{12}$ was decreased $\left(0.25\right.$ or $0.5 \mathrm{~mol} \%$ ), where $66 \%$ and $52 \%$ of $\mathrm{Et}_{3} \mathrm{SiH}$ was found to be unreacted ( $\mathrm{GC}$ ratio), even after $12 \mathrm{~h}$ at $100^{\circ} \mathrm{C}$ (Table 1 , Runs 1 and 2).

The reaction went more slowly when carried out at $20{ }^{\circ} \mathrm{C}, 40$ ${ }^{\circ} \mathrm{C}, 60{ }^{\circ} \mathrm{C}$ or $80{ }^{\circ} \mathrm{C}$. Thus, significant amount of $\mathrm{Et}_{3} \mathrm{SiH}$ was found to be unreacted $\left(82 \%\right.$ at $40{ }^{\circ} \mathrm{C}, 70 \%$ at $60{ }^{\circ} \mathrm{C}, 61 \%$ at 80 ${ }^{\circ} \mathrm{C}$ ) even being heated for $24 \mathrm{~h}$ (Table 1, Runs 10-12). No silyl ester was detected at $20^{\circ} \mathrm{C}$ even after $24 \mathrm{~h}$ and all of the $\mathrm{Et}_{3} \mathrm{SiH}$ was recovered (Table 1, Run 9). When the amount of EtI was increased to $8 \mathrm{~mol} \%$, the product yields were $94 \%$ (Table 1 ,
Run 7). The reaction went more slowly when the amount of EtI was decreased to $2 \mathrm{~mol} \%$, where $22 \%$ of $^{2} t_{3} \mathrm{SiH}$ remained unreacted, even after $12 \mathrm{~h}$ at $100{ }^{\circ} \mathrm{C}$ (Table 1, Run 8).

Dehydrosilylation in different solvents was also investigated (Table 2). In xylene, ethylbenzene, tert-butylbenzene, mesitylene, $n$-octane, diethylene glycol diethyl ether, and anisole, the dehydrogenative coupling is slightly slower compared with toluene, and some amount of $\mathrm{Et}_{3} \mathrm{SiH}$ was detected (12-22\%) (Table 2, Runs 2-8). In N,N-dimethylformamide (DMF) and $N, N$-dimethylacetamide (DMAc), the dehydrocoupling failed to reach completion being heated at $100{ }^{\circ} \mathrm{C}$ for $48 \mathrm{~h}$, and significant amounts of $\mathrm{Et}_{3} \mathrm{SiH}$ were detected (Table 2, Runs 9 and 10).

Other Ru complexes were also tested as catalysts for the dehydrogenative coupling and the results were summarized in Table 3 (usually at $1 \mathrm{~mol} \%$ of Ru complex, $4 \mathrm{~mol} \%$ EtI, toluene, 100 $\left.{ }^{\circ} \mathrm{C}\right)$. In the case of using $\left[\mathrm{RuCl}_{2}(\mathrm{CO})_{3}\right]_{2}, \mathrm{RuCl}_{2}(\mathrm{CO})_{2}\left(\mathrm{PPh}_{3}\right)_{2}$, $\mathrm{Ru}(\mathrm{acac})_{3}, \mathrm{RuCl}_{2}\left(2,2^{\prime} \text {-bipy }\right)_{3}$ and $\mathrm{RuCl}_{2}\left(\mathrm{PPh}_{3}\right)_{3}$ as catalysts, $9-27 \%$ of $\mathrm{Et}_{3} \mathrm{SiH}$ was still detected after heating for $24 \mathrm{~h}$ at 


\begin{tabular}{|c|c|c|c|c|c|}
\hline Run & Acid & Silane & Time (h) & Product & Yield $(\%)^{b}$ \\
\hline 1 & $\mathrm{CH}_{3} \mathrm{CO}_{2} \mathrm{H}$ & $\mathrm{Et}_{3} \mathrm{SiH}$ & 8 & $\mathrm{CH}_{3} \mathrm{CO}_{2} \mathrm{SiEt}_{3}$ & $94[30]$ \\
\hline 2 & $\mathrm{CH}_{3} \mathrm{CO}_{2} \mathrm{H}$ & $(n-\mathrm{Pr})_{3} \mathrm{SiH}$ & 8 & $\mathrm{CH}_{3} \mathrm{CO}_{2} \mathrm{SiPr}_{3}{ }_{3}$ & $92[48]$ \\
\hline 3 & $\mathrm{CH}_{3} \mathrm{CO}_{2} \mathrm{H}$ & $(n-\mathrm{Bu})_{3} \mathrm{SiH}$ & 8 & $\mathrm{CH}_{3} \mathrm{CO}_{2} \mathrm{SiBu}_{3}{ }_{3}$ & $93[48]$ \\
\hline 4 & $\mathrm{CH}_{3} \mathrm{CH}_{2} \mathrm{CO}_{2} \mathrm{H}$ & $\mathrm{Et}_{3} \mathrm{SiH}$ & 8 & $\mathrm{CH}_{3} \mathrm{CH}_{2} \mathrm{CO}_{2} \mathrm{SiEt}_{3}$ & $95[28]$ \\
\hline 5 & $\mathrm{CH}_{3} \mathrm{CH}_{2} \mathrm{CO}_{2} \mathrm{H}$ & $(n-\mathrm{Pr})_{3} \mathrm{SiH}$ & 8 & $\mathrm{CH}_{3} \mathrm{CH}_{2} \mathrm{CO}_{2} \mathrm{SiPr}_{3}{ }_{3}$ & $89[48]$ \\
\hline 6 & $\mathrm{CH}_{3} \mathrm{CH}_{2} \mathrm{CO}_{2} \mathrm{H}$ & $(n-\mathrm{Bu})_{3} \mathrm{SiH}$ & 8 & $\mathrm{CH}_{3} \mathrm{CH}_{2} \mathrm{CO}_{2} \mathrm{SiBu}_{3}{ }_{3}$ & $91[49]$ \\
\hline 7 & $\mathrm{CH}_{3}\left(\mathrm{CH}_{2}\right)_{8} \mathrm{CO}_{2} \mathrm{H}$ & $(\text { iso-Pr })_{3} \mathrm{SiH}$ & 9 & $\mathrm{CH}_{3}\left(\mathrm{CH}_{2}\right)_{8} \mathrm{CO}_{2} \mathrm{SiPr}_{3}^{\mathrm{i}}$ & $90[18]$ \\
\hline 8 & $\mathrm{C}_{6} \mathrm{H}_{5} \mathrm{CH}_{2} \mathrm{CO}_{2} \mathrm{H}$ & $\mathrm{Et}_{3} \mathrm{SiH}$ & 8 & $\mathrm{C}_{6} \mathrm{H}_{5} \mathrm{CH}_{2} \mathrm{CO}_{2} \mathrm{SiEt}_{3}$ & $92[28]$ \\
\hline 9 & $\mathrm{C}_{6} \mathrm{H}_{5} \mathrm{CH}_{2} \mathrm{CO}_{2} \mathrm{H}$ & $(\text { iso-Pr })_{3} \mathrm{SiH}$ & 9 & $\mathrm{C}_{6} \mathrm{H}_{5} \mathrm{CH}_{2} \mathrm{CO}_{2} \mathrm{SiPr}_{3}^{\mathrm{i}}$ & $93[18]$ \\
\hline 10 & $\mathrm{C}_{6} \mathrm{H}_{5} \mathrm{CH}_{2} \mathrm{CO}_{2} \mathrm{H}$ & tert-BuMe ${ }_{2} \mathrm{SiH}$ & 10 & $\mathrm{C}_{6} \mathrm{H}_{5} \mathrm{CH}_{2} \mathrm{CO}_{2} \mathrm{SiMe}_{2} \mathrm{Bu}^{t}$ & $85[18]$ \\
\hline 11 & $\mathrm{C}_{6} \mathrm{H}_{5} \mathrm{CH}(\mathrm{Me}) \mathrm{CO}_{2} \mathrm{H}$ & $(\text { iso-Pr })_{3} \mathrm{SiH}$ & 9 & $\mathrm{C}_{6} \mathrm{H}_{5} \mathrm{CH}(\mathrm{Me}) \mathrm{CO}_{2} \mathrm{SiPr}_{3}{ }_{3}$ & $92[18]$ \\
\hline 12 & $3-\mathrm{BrC}_{6} \mathrm{H}_{4} \mathrm{CO}_{2} \mathrm{H}$ & (iso-Pr) ${ }_{3} \mathrm{SiH}$ & 8 & $3-\mathrm{BrC}_{6} \mathrm{H}_{4} \mathrm{CO}_{2} \mathrm{SiPr}_{3}^{\mathrm{i}}$ & $92[50]$ \\
\hline 13 & $3-\mathrm{BrC}_{6} \mathrm{H}_{4} \mathrm{CO}_{2} \mathrm{H}$ & tert-BuMe ${ }_{2} \mathrm{SiH}$ & 10 & $3-\mathrm{BrC}_{6} \mathrm{H}_{4} \mathrm{CO}_{2} \mathrm{SiMe}_{2} \mathrm{Bu}^{t}$ & $86[50]$ \\
\hline 14 & $3-\mathrm{ClC}_{6} \mathrm{H}_{4} \mathrm{CO}_{2} \mathrm{H}$ & (iso-Pr) ${ }_{3} \mathrm{SiH}$ & 8 & $3-\mathrm{ClC}_{6} \mathrm{H}_{4} \mathrm{CO}_{2} \mathrm{SiPr}_{3}{ }_{3}$ & $91[50]$ \\
\hline 15 & $3-\mathrm{ClC}_{6} \mathrm{H}_{4} \mathrm{CO}_{2} \mathrm{H}$ & tert-BuMe ${ }_{2} \mathrm{SiH}$ & 10 & $3-\mathrm{ClC}_{6} \mathrm{H}_{4} \mathrm{CO}_{2} \mathrm{SiMe}_{2} \mathrm{Bu}^{t}$ & $85[50]$ \\
\hline 16 & $4-\mathrm{ClC}_{6} \mathrm{H}_{4} \mathrm{CO}_{2} \mathrm{H}$ & $\mathrm{Et}_{3} \mathrm{SiH}$ & 8 & $4-\mathrm{ClC}_{6} \mathrm{H}_{4} \mathrm{CO}_{2} \mathrm{SiEt}_{3}$ & $91[51]$ \\
\hline 17 & $\mathrm{C}_{6} \mathrm{H}_{5} \mathrm{CO}_{2} \mathrm{H}$ & $\mathrm{Et}_{3} \mathrm{SiH}$ & 8 & $\mathrm{C}_{6} \mathrm{H}_{5} \mathrm{CO}_{2} \mathrm{SiEt}_{3}$ & $93[52]$ \\
\hline 18 & $\mathrm{C}_{6} \mathrm{H}_{5} \mathrm{CO}_{2} \mathrm{H}$ & $(n-\mathrm{Pr})_{3} \mathrm{SiH}$ & 8 & $\mathrm{C}_{6} \mathrm{H}_{5} \mathrm{CO}_{2} \mathrm{SiPr}_{3}{ }_{3}$ & $92[53]$ \\
\hline 19 & $\mathrm{C}_{6} \mathrm{H}_{5} \mathrm{CO}_{2} \mathrm{H}$ & $(\text { iso- } \mathrm{Pr})_{3} \mathrm{SiH}$ & 9 & $\mathrm{C}_{6} \mathrm{H}_{5} \mathrm{CO}_{2} \mathrm{SiPr}_{3}^{\mathrm{i}}$ & $95[18]$ \\
\hline 20 & $\mathrm{C}_{6} \mathrm{H}_{5} \mathrm{CO}_{2} \mathrm{H}$ & $(n-\mathrm{Bu})_{3} \mathrm{SiH}$ & 8 & $\mathrm{C}_{6} \mathrm{H}_{5} \mathrm{CO}_{2} \mathrm{SiBu}_{3}{ }_{3}$ & $91[54]$ \\
\hline 21 & & $\mathrm{Et}_{3} \mathrm{SiH}$ & 8 & & $94[55]$ \\
\hline 22 & & $\left(\right.$ iso-Pr) ${ }_{3} \mathrm{SiH}$ & 9 & & $93[18]$ \\
\hline 23 & $4-\mathrm{O}_{2} \mathrm{NC}_{6} \mathrm{H}_{4} \mathrm{CO}_{2} \mathrm{H}$ & tert-BuMe ${ }_{2} \mathrm{SiH}$ & 10 & $4-\mathrm{O}_{2} \mathrm{NC}_{6} \mathrm{H}_{4} \mathrm{CO}_{2} \mathrm{SiMe}_{2} \mathrm{Bu}^{t}$ & 88 [56] \\
\hline
\end{tabular}

${ }^{a}$ Carboxylic acid $(20 \mathrm{mmol})$, silane $(20 \mathrm{mmol}), \mathrm{Ru}_{3}(\mathrm{CO}){ }_{12}(0.2 \mathrm{mmol}, 1 \mathrm{~mol} \%)$, Etl $(4 \mathrm{~mol} \%), 100{ }^{\circ} \mathrm{C}$. blsolated yield.

$100{ }^{\circ} \mathrm{C}$ (Table 3, Runs 4-8). In the presence of $4 \mathrm{~mol} \%$ of ethyl bromide, the dehydrogenation was slightly slower compared with EtI, and a small amount of $\mathrm{Et}_{3} \mathrm{SiH}$ was still found. Without EtI, the dehydrocoupling was sluggish and $81 \%$ of $\mathrm{Et}_{3} \mathrm{SiH}$ were detected even being heated for $24 \mathrm{~h}$ at $100{ }^{\circ} \mathrm{C}$ (Table 3, Run 3).

Treatment of a number of carboxylic acids and silanes such as triethylsilane, tri- $n$-propylsilane $\left(n-\mathrm{Pr}_{3} \mathrm{SiH}\right)$, tri-iso-propylsilane (iso- $\left.\mathrm{Pr}_{3} \mathrm{SiH}\right)$, tri- $n$-butylsilane $\left(n-\mathrm{Bu}_{3} \mathrm{SiH}\right)$ or tertbutyldimethylsilane (tert-BuMe $2 \mathrm{SiH}$ ) afforded the corresponding silyl esters in good and excellent yields (all with 1 $\mathrm{mol} \% \mathrm{Ru}_{3}(\mathrm{CO})_{12}$ and $4 \mathrm{~mol} \% \mathrm{EtI}$ in toluene at $100{ }^{\circ} \mathrm{C}$, Table 4). In the case of nitro-, bromo- and chlorobenzoic acid, the expected silyl esters were obtained in $85-95 \%$ yields, free of dehalogenated or over-reduced by-products (Table 4, Runs 12-16 and 23).

\section{Conclusion}

In conclusion, we have demonstrated that $\mathrm{Ru}_{3}(\mathrm{CO})_{12} / \mathrm{EtI}$ is an efficient catalytic system for the dehydrogenative cross-coup- ling of carboxylic acids with silanes. The dehydrogenative cross-coupling reactions proceed efficiently to give the corresponding silyl esters in good and excellent yields. No overreduced silyl esters are formed in the case of coupling nitro-, bromo-, and chlorobenzoic acid with silanes. We believe that the $\mathrm{Ru}_{3}(\mathrm{CO})_{12} /$ EtI-catalyzed dehydrosilylation of carboxylic acids with silanes provides another important protocol for a one-step, highly selective, atom-economical and efficient synthetic method. We are currently broadening the scope of this dehydrosilylation of carboxylic acids and silanes in our laboratory and the results will be published elsewhere.

\section{Experimental}

To a mixture of propionic acid (40 mmol, $2.96 \mathrm{~g}$ ), and triethylsilane $(40 \mathrm{mmol}, 4.64 \mathrm{~g})$ in toluene $(20 \mathrm{ml})$ was added $\mathrm{Ru}_{3}(\mathrm{CO})_{12}(0.4 \mathrm{mmol}, 0.01$ equiv $)$ and EtI ( $2.0 \mathrm{mmol}, 0.05$ equiv) at room temperature under a nitrogen atmosphere. The reaction mixture was stirred at $100{ }^{\circ} \mathrm{C}$ for 8 hours (monitored by GC). The desired triethylsilyl propionate was obtained as a colourless oil (yield: 95\%) after distillation under reduced pres- 
sure (Table 1, Run 3). Triethylsilyl propionate [29]: IR (neat): 686, 742, 826, 995, 1063, 1240, 1410, 1466, 1718, 2872, $2952 \mathrm{~cm}^{-1}$. ${ }^{1} \mathrm{H}$ NMR (400 MHz, $\left.\mathrm{CDCl}_{3}\right): \delta 0.74\left(6 \mathrm{H}, \mathrm{q},{ }^{3} J 7.8\right.$ $\mathrm{Hz}), 0.95\left(9 \mathrm{H}, \mathrm{t},{ }^{3} J 7.8 \mathrm{~Hz}\right), 1.14\left(3 \mathrm{H}, \mathrm{t},{ }^{3} J 7.6 \mathrm{~Hz}\right), 2.36(2 \mathrm{H}, \mathrm{q}$, $\left.{ }^{3} J 7.6 \mathrm{~Hz}\right) .{ }^{13} \mathrm{C}$ NMR $\left(100 \mathrm{MHz}, \mathrm{CDCl}_{3}\right): 4.46,6.48,9.32$, 28.44, 175.26 .

All of the silyl esters are known compounds and were compared with authentic samples [prepared by cross-coupling of carboxylic acids and chlorosilanes in the presence of a base such as triethylamine or imidazole (tert-butylsilyl esters) in dichloromethane] and were identified on the basis of their IR, ${ }^{1} \mathrm{H}$ NMR, ${ }^{13} \mathrm{C}$ NMR and GC-MS spectral data.

\section{References}

1. Cazacu, M.; Munteanu, G.; Racles, C.; Vlad, A.; Marcu, M. J. Organomet. Chem. 2006, 691, 3700-3707. doi:10.1016/ j.jorganchem.2006.05.026

2. Peppas, N. A.; Langer, R. Science 1994, 263, 1715-1720. doi:10.1126/ science. 8134835

3. Langer, R. Acc. Chem. Res. 1993, 26, 537-542. doi:10.1021/ ar00034a004

4. Swift, G. Acc. Chem. Res. 1993, 26, 105-110. doi:10.1021/ ar00027a005

5. Wang, M.; Gan, D.; Wooley, K. L. Macromolecules 2001, 34, 3215-3223. doi:10.1021/ma0100450

6. Lim, Y.; Choi, Y. H.; Park, J. J. Am. Chem. Soc. 1999, 121, 5633-5639. doi:10.1021/ja984012k

7. Wang, M.; Weinberg, J. M.; Wooley, K. L. Macromolecules 1998, 31 , 7606-7612. doi:10.1021/ma9808981

8. Penco, M.; Marcioni, S.; Ferruti, P.; D'Antone, S.; Deghenghi, R. Biomaterials 1996, 17, 1583-1590. doi:10.1016/0142-9612(95)00323-1

9. Haslam, E. Tetrahedron 1980, 36, 2409-2433. doi:10.1016/00404020(80)80219-5

10. Cunico, R. F.; Bedell, L. J. Org. Chem. 1980, 45, 4797-4798. doi:10.1021/jo01311a058

11. Gilding, D. K.; Reed, A. M. Polymer 1979, 20, 1459-1464. doi:10.1016/ 0032-3861(79)90009-0

12. Kayan, A.; Hoebbel, D.; Schmidt, H. J. Appl. Polym. Sci. 2005, 95, 790-796. doi:10.1002/app.21315

13. Jaoui, M.; Kleindienst, T. E.; Lewandowski, M.; Edney, E. O. Anal. Chem. 2004, 76, 4765-4778. doi:10.1021/ac049919h

14. Pierce, A. E. Silylation of Organic Compounds; Pierce Chem. Co.: Rockford, Illinois, 1968.

15. Greene, T. W.; Wuts, P. G. M. Protective Groups in Organic Synthesis, 3rd ed.; Wiley and Sons: New York, 1999.

16. Kocieński, P. J. Protecting Groups; George Thieme Verlag: New York, 1999.

17. Oyama, K.; Kondo, T. Org. Lett. 2003, 5, 209-212. doi:10.1021/ ol027263d

18. Lee, A. S.-Y.; Su, F.-Y. Tetrahedron Lett. 2005, 46, 6305-6309. doi:10.1016/j.tetlet.2005.07.049

19. Heldmann, D. K.; Stohrer, J.; Zauner, R. Synlett 2002, 1919-1921. doi:10.1055/s-2002-34898

20. Uchiyama, N.; Akiyama, H. Jpn. Kokai Tokkyo Koho JP 200467579, 2004.

Chem. Abstr. 2004, 140, 199907.
21. Masuoka, S.; Ito, M.; Honda, Y. Production of polymerizable monomer. JP 04342593 A, November 30, 1992. Chem. Abstr. 1993, 118, 192474.

22. Mbah, G. C.; Speier, J. L. J. Organomet. Chem. 1984, 271, 77-82. doi:10.1016/0022-328X(84)85165-7

23. Eaborn, C.; Happer, D. A. R.; Hopper, S. P.; Safa, K. D. J. Organomet. Chem. 1980, 188, 179-192. doi:10.1016/S0022-328X(00)82811-9

24. Jaspars, M. Silyl Esters. In Compounds of Group 15 (As, Sb, Bi) and Silicon Compounds; Fleming, I., Ed.; Science of Synthesis, Vol. 4; Thieme: Stuttgart, 2001; pp 293-304.

For a review.

25. Masaoka, S.; Banno, T. Jpn. Kokai Tokkyo Koho JP 2004182681, 2004.

Chem. Abstr. 2004, 141, 54484.

26. Kubota, Y.; Kubota, T. Jpn. Kokai Tokkyo Koho JP 2004210644, 2004. Chem. Abstr. 2004, 141, 140615.

27. Gauvin, F.; Harrod, J. F.; Woo, H. G. Catalytic Dehydrocoupling: A General Strategy for the Formation of Element-Element Bonds. In Advances in Organometallic Chemistry; Stone, F. G. A.; West, R., Eds.; Academic Press: New York, 1998; Vol. 42.

28. Huang, X.; Craita, C.; Awad, L.; Vogel, P. Chem. Commun. 2005, 1297-1299. doi:10.1039/b417894g

29. Liu, G.-B. Synlett 2006, 1431-1433. doi:10.1055/s-2006-941576

30. Chauhan, M.; Chauhan, B. P. S.; Boudjouk, P. Org. Lett. 2000, 2, 1027-1029. doi:10.1021/ol005507t

31. Li, Y.; Kawakami, Y. Macromolecules 1999, 32, 8768-8773. doi:10.1021/ma991312t

32. lovel, I.; Popelis, J.; Gaukhman, A.; Lukevics, E. J. Organomet. Chem. 1998, 559, 123-130. doi:10.1016/S0022-328X(98)00431-8

33. Fujino, J.; Taniguchi, H.; Mori, K.; Funaoka, S.; Ito, M. Jpn. Kokai Tokkyo Koho JP 10212293, 1998. Chem. Abstr. 1998, 129, 216765.

34. Schubert, U.; Lorenz, C. Inorg. Chem. 1997, 36, 1258-1259. doi:10.1021/ic961134+

35. Masuoka, S.; Ito, M.; Honda, Y. Jpn. Kokai Tokkyo Koho JP 05025187, 1993. Chem. Abstr. 1993, 119, 75081.

36. Masuoka, S.; Ito, M.; Honda, Y. Jpn. Kokai Tokkyo Koho JP 04342593 , 1992.

Chem. Abstr. 1993, 118, 192474.

37. Aizpurua, J. M.; Palomo, C.; Palomo, A. L. Can. J. Chem. 1984, 62, 336-340. doi:10.1139/v84-059

38. Aizpurua, J. M.; Palomo, C. Bull. Soc. Chim. Fr. 1982, II-265-II-268.

39. Morita, T.; Okamoto, Y.; Sakurai, H. Synthesis 1981, 745-746. doi:10.1055/s-1981-29588

40. Morita, T.; Okamoto, Y.; Sakurai, H. Tetrahedron Lett. 1980, 21 , 835-838. doi:10.1016/S0040-4039(00)71518-8

41. Palomo, C. Synthesis 1981, 809-811. doi:10.1055/s-1981-29606

42. Haslam, E. Tetrahedron 1980, 36, 2409-2433. doi:10.1016/00404020(80)80219-5

43. Sauer, R. O.; Patnode, W. J. Am. Chem. Soc. 1945, 67, 1548-1549. doi:10.1021/ja01225a046

44. Yamamoto, K.; Takemae, M. Bull. Chem. Soc. Jpn. 1989, 62, 2111-2113. doi:10.1246/bcsj.62.2111

45. Corriu, R. J. P.; Moreau, J. J. E.; Praet, H. Organometallics 1990, 9, 2086-2091. doi:10.1021/om00157a017

46. Liu, G.-B.; Zhao, H.-Y.; Thiemann, T. Synth. Commun. 2007, 37, 2717-2727. doi:10.1080/00397910701465669

47. Liu, G.-B.; Zhao, H.-Y.; Thiemann, T. Adv. Synth. Catal. 2007, 349, 807-811. doi:10.1002/adsc.200600338 
48. Prince, R. H.; Timms, R. E. Inorg. Chim. Acta 1968, 2, 260-262. doi:10.1016/S0020-1693(00)87038-3

49. Masuoka, S.; Matsubara, Y.; Mori, K.; Oka, M. Antifouling coating material, antifouling coating film, submerged structure, and antifouling method. PCT Int. Appl. WO 01/81489 A1, November 1, 2001. Chem. Abstr. 2001, 135, 345937.

50. Iranpoor, N.; Firouzabadi, H.; Shaterian, H. R. Synth. Commun. 2002, 32, 3653-3657. doi:10.1081/SCC-120014983

51. Orlov, N. F.; Slesar, L. N. J. Gen. Chem. USSR 1966, 36, 1093-1097.

52. Voronkov, M. G.; Pukhnarevich, V. B.; Ushakova, N. I.; Burnashova, T. D.; Tsykhanskaya, I. I.; Koval'skaya, N. B. Zh. Obshch. Khim. 1990, 60, 1584-1587.

Chem. Abstr. 1991, 114, 81153.

53. Frainnet, É.; Paul, M. C. R. Seances Acad. Sci., Ser. C 1967, 265, 1185-1188.

Chem. Abstr. 1968, 68, 59653.

54. Biryukov, I. P.; Khudobin, Yu. I.; Kharitonov, N. P.; Yazov, A. N. Zh. Fiz. Khim. 1977, 51, 2111-2113. Chem. Abstr. 1977, 87, 167048.

55. Firouzabadi, H.; Iranpoor, N.; Shaterian, H. R. Phosphorus, Sulfur Silicon Relat. Elem. 2000, 166, 71-81. doi:10.1080/ 10426500008076532

56. Mai, K.; Patil, G. J. Org. Chem. 1986, 51, 3545-3548. doi:10.1021/ jo00368a030

\section{License and Terms}

This is an Open Access article under the terms of the Creative Commons Attribution License

(http://creativecommons.org/licenses/by/2.0), which permits unrestricted use, distribution, and reproduction in any medium, provided the original work is properly cited.

The license is subject to the Beilstein Journal of Organic Chemistry terms and conditions:

(http://www.beilstein-journals.org/bjoc)

The definitive version of this article is the electronic one which can be found at: doi:10.3762/bjoc. 4.27 